https://doi.org/10.47660/CBR.2020.16855

\title{
NEUROSYPHILIS MIMICKING ANCA ASSOCIATED VASCULITIS
}

Flavio Barboza ${ }^{1,{ }^{*}}$, Heloisa Maria Lopes Scarinci ${ }^{1}$, Evelyn Angrevski Rodrigues ${ }^{1}$, Talles Henrique Pichinelli Maffei ${ }^{1}$, Ygor Augusto Silva Lima ${ }^{1}$, Lucas do Carmo de Carvalho ${ }^{1}$, Thalyne Aparecida Leite de Lima ${ }^{1}$, Nohati Rhanda Freitas dos Santos ${ }^{1}$, Bruna Luiza Oliveira Lima ${ }^{1}$, Bruna Sayuri Tanaka ${ }^{1}$, Raquel Gerep Pereira ${ }^{1}$, José Belúcio Neto²

1. Universidade Federal do Mato Grosso, Sinop (MT), Brazil. 2. Universidade Federal de São Paulo, São Paulo (SP), Brazil.

*Corresponding author: flavio.fbarboza@uol.com.br

\section{BACKGROUND}

Associated or pauci-immune antineutrophil cytoplasmic antibody (ANCA) vasculitis, represented by Wegener's granulomatosis, microscopic polyangiitis and Churg-Strauss syndrome, affect small-caliber vessels. They can cause lung-kidney syndrome (rapidly progressive glomerulonephritis and hemoptysis), uveitis, recurrent sinusitis, perforations of the upper airways, hearing loss, palpable purpura, polyarthralgia, neuropathy, among others. These are rare diseases, therefore, the exclusion of differential diagnoses, including syphilis, is mandatory. The ocular, meningeal and auditory involvement associated with fever, skin lesions and joint pain, compatible with neurosyphilis and its systemic manifestations, is the main highlight.

\section{CASE REPORT}

A 61-year-old woman reported nodules in the neck, nasal obstruction and fever, followed by an otolaryngologist. Videolaryngoscopic investigation, finding of an obstructive mass that was referred for biopsy (nasal polyposis with intense eosinophilic infiltrate) evolved with bilateral deafness, eye pain and involuntary weight loss (7 kg in 3 months). On physical examination, cervical lymph node enlargement, polyarthritis of the wrists, knees, ankles and metacarpal phalanges, palpable purpura in the upper and lower limbs and afebrile ocular hyperemia. After ophthalmic evaluation, diagnosis of bilateral panuveitis, attempted treatment with topical corticosteroids, with no response. Corticotherapy was started for uveitis and referred to a rheumatologist for investigation of associated ANCA vasculitis. Prednisone $1 \mathrm{mg} / \mathrm{kg}$ showed eye and joint improvement, but not auditory. Tests performed showed increased evidence of inflammatory activity (ESR, CRP), nuclear speckled FAN 1:80, ANCA negative, normal creatinine and urea, absence of proteinuria or urinary erythrocyte dysmorphism, hepatitis B, C serology, HIV negative, VDRL positive (1:256) and confirmatory test with positive FTA ABS. After the diagnosis of neurosyphilis, due to the impossibility of performing CSF and the beginning of penicillin due to lack of availability in the country, the treatment instituted was ceftriaxone EV 15 days. Monitoring the VDRL every 15 days reveals 1:64, 1:32, 1: 8, after two months of treatment, VDRL 1:4 with better complete symptoms. The slide review of the initial biopsy of nasal polyp, histopathological compatible with syphilitic gum.

\section{CONCLUSION}

Faced with conditions such as uveitis, deafness, nasal polyposis, arthritis and palpable purple, which suggest vasculitic conditions, infections cannot be excluded. Syphilis, an endemic disease that has reemerged in the recent years in Brazil, and that can mimic associated ANCA vasculitis, should always be investigated. 